John C. Lieske*, Olga Bondar, W. Greg Miller, Lorin M. Bachmann, Andrew S. Narva, Yoshihisa Itoh, Ingrid Zegers, Heinz Schimmel, Karen Phinney and David M. Bunk on behalf of the National Kidney Disease Education Program-IFCC Working Group on Standardization of Albumin in Urine (WG-SAU)

\title{
A reference system for urinary albumin: current status
}

\section{Abstract}

Background: Increased urinary excretion of albumin reflects kidney damage and is a recognized risk factor for progression of renal and cardiovascular disease. Considerable inter-method differences have been reported for both albumin and creatinine measurement results, and therefore the albumin-to-creatinine ratio. Measurement accuracy is unknown and there are no independent reference measurement procedures for albumin and no reference materials for either measurand in urine.

Methods: The National Kidney Disease Education Program (NKDEP) Laboratory Working Group and the International Federation of Clinical Chemistry and Laboratory Medicine (IFCC) have initiated joint projects to facilitate standardization of urinary albumin and creatinine measurement.

Results: A candidate LC-MS/MS reference measurement procedure for urinary albumin and candidate reference materials for urinary albumin and creatinine has been developed. The status of validations of these reference system components is reported.

Conclusions: The development of certified reference materials and reference measurement procedures for urinary albumin will enable standardization of this important measurand.

Keywords: chronic kidney disease; microalbumin; reference material; reference method; urine albumin.

\footnotetext{
*Corresponding author: John C. Lieske, Mayo Clinic Renal Function Laboratory, Department of Laboratory Medicine and Pathology, Mayo Clinic Division of Nephrology and Hypertension, Department of Internal Medicine, Rochester, MN 55905, USA,

E-mail: Lieske.John@mayo.edu

Olga Bondar: Mayo Clinic Renal Function Laboratory, Department of Laboratory Medicine and Pathology, Mayo Clinic Division of Nephrology and Hypertension, Department of Internal Medicine, Rochester, MN, USA

W. Greg Miller and Lorin M. Bachmann: Department of Pathology, Virginia Commonwealth University, Richmond, VA, USA

Andrew S. Narva: National Kidney Disease Education Program, National Institute for Diabetes and Digestive Diseases, National Institutes of Health, Bethesda, MD, USA
}

Yoshihisa Itoh: Department of Laboratory Medicine, Asahikawa Medical College, Asahikawa, Japan

Ingrid Zegers and Heinz Schimmel: European Commission, Joint Research Centre, Institute for Reference Materials and Measurements (IRMM), Geel, Belgium

Karen Phinney and David M. Bunk: Analytical Chemistry Division, National Institute of Standards and Technology, Gaithersburg, MD, USA

\section{Introduction}

Increased urinary excretion of albumin reflects kidney damage and is a widely recognized risk factor for progression of renal and cardiovascular disease (CVD). Considerable inter-method differences have been reported for both albumin and creatinine measurement procedures [and, therefore, the albumin-to-creatinine ratio (ACR)], but accuracy is unknown and there are no independent reference measurement procedures for albumin and no reference materials for either measurand in urine [1] At present most IVD manufacturers provide calibrators with values that are traceable either to the serum reference material ERM-DA470k/IFCC, certified for its albumin content by immunoassay [2], or to purified albumin preparations. Only recently a highly purified human serum albumin reference material was prepared specifically for the standardization of urinary albumin measurements [3].

The National Kidney Disease Education Program (NKDEP) Laboratory Working Group and the International Federation of Clinical Chemistry and Laboratory Medicine (IFCC) have initiated joint projects to facilitate standardization of urinary albumin measurement. Over the last 4 years much progress has been made towards development of a candidate reference measurement procedure for urinary albumin that employs liquid chromatography coupled to tandem mass spectrometry (LC-MS/MS), and preparation and evaluation of urinary albumin and creatinine reference materials. A large-scale assessment of harmonization among routine measurement procedures was also initiated which aims to evaluate the comparability of 
urinary albumin measurement procedures, the effects of interferences, and the suitability of existing (serum) reference materials and candidate reference materials. This report describes the status of these efforts.

\section{Results}

\section{Initial impressions of agreement among routine measurement procedures and commutability of diluted ERM-DA470k/IFCC}

To facilitate the development of recommendations for standardization of routine urinary albumin measurement procedures, the joint Working Group identified the following goals: 1) obtain and evaluate objective data on the current state of agreement among different routine measurement procedures for urinary albumin; 2) identify potential urine matrix and albumin forms for which routine measurement procedures are expected to provide good performance; 3) evaluate the equivalence of immunoassay measurement procedures based on comparison with the candidate LC-MS/MS reference measurement procedure; and 4) evaluate the commutability characteristics of two candidate reference materials for urinary albumin (highly-purified HSA from the Japanese Society of Clinical Chemistry and dilutions of ERM-DA470k/IFCC), to determine the effectiveness of these materials for standardization efforts.

To address these goals, 342 residual native urine samples were obtained from patients for whom urinary albumin measurements were made as part of their routine medical care. The fresh urine samples were shipped overnight at $2-8^{\circ} \mathrm{C}$ for testing by 16 commercially available quantitative routine urinary albumin measurement procedures. A series of centrally-prepared dilutions of ERMDA470k/IFCC were also distributed to participants for blinded analysis with patient samples. In addition, each participant prepared dilutions of the ERM-DA470k/IFCC and the JSCC reference materials.

Preliminary evaluation of agreement among routine measurement procedures suggested that medians of the measured concentrations of patient urine samples varied approximately $40 \%$ for urinary albumin concentrations of $12-1270 \mathrm{mg} / \mathrm{L}$. Preliminary visual inspection of the relationship of results between the routine measurement procedures and the candidate reference measurement procedure suggested that diluted ERM-DA470k material was likely to be commutable with patient results for at least 11 of 16 routine measurement procedures. The commutability characteristics of the ERM-DA470k materials for the remaining five methods were indeterminate by visual inspection. The data generated from this study will be analyzed in detail and used to determine the feasibility of improving standardization of results among routine measurement procedures through use of currently available reference materials and the candidate reference measurement procedure.

\section{Urinary albumin and creatinine certified reference materials (CRMs)}

\section{ERM-DA470 and ERM-DA470k/IFCC}

Most routine measurement procedures for albumin in serum produce results that are traceable to the certified reference material ERM-DA470k/IFCC or to its predecessor ERM-DA470. ERM-DA470 was produced in the early 1990s after extensive feasibility studies [4]. The result was a material that, although extensively stabilized, delipidated and freeze-dried, had properties close enough to serum samples to be commutable for most proteins that were value assigned. Quantity values were assigned to the material with routine clinical laboratory immunoassays that were further optimized. In 2008 this batch of material was exhausted and ERM-DA470k/IFCC was released to replace it. The value for albumin in ERM-DA40k/IFCC was again assigned by immunoassays, using ERM-DA470 as calibrator [2].

These reference materials have been used by a majority of manufacturers for assigning values to their product calibrators for routine serum measurement procedures. External quality assessment schemes show that in general measurements of serum albumin are under control, with between-laboratory coefficient of variations (CVs) around 5\%-6\% for all measurement procedures, including methods based on turbidimetry, nephelometry, and the use of dye-binding chromophores [results from the Referenzinstitut für Bioanalytik (http:// www.dgkl-rfb.de/) in 2011 and 2012]. The success of ERM-DA470 for serum measurements is to a considerable extent due to the development of very carefully controlled procedures for transferring values from the CRM to manufacturers' master calibrators and from master calibrators to product calibrators [5]. There are still specific cases in which a significant bias between measurement procedures exists, and it has been postulated that this bias could be due to problems with the transfer of values along the traceability chain [6] or to use of different dye binding chromophores. 
The use of ERM-DA470k/IFCC for the calibration of urinary albumin measurements requires that dilutions of the CRM are commutable for the different measurement procedures to be calibrated. Such dilutions are not necessarily trivial, as the urine matrix is quite different from diluted serum. Therefore, the commutability of dilutions of ERM-DA470k/IFCC for urinary albumin measurements is the subject of a large-scale commutability study (discussed previously). If dilutions prepared according to controlled protocols are found to be sufficiently commutable, their use for calibration may lead to harmonized urinary albumin results.

\section{Pure HSA CRM}

NIST is in the process of developing a primary certified reference material for albumin (NIST SRM 2925, 'Human serum albumin solution'). The intended use of this certified reference material will be the preparation of calibration solutions for the reference measurement procedure of albumin in human urine. Recombinant human serum albumin (rHSA) to prepare this CRM has been purchased from a commercial source and evaluated at NIST to assess protein purity and molecular structure heterogeneity. The target albumin concentration of NIST SRM 2925 is approximately $1 \mathrm{~g} / \mathrm{L}$. NIST SRM 2925 will be supplied in approximately $1 \mathrm{~mL}$ aliquots of frozen solution, stored at $-80^{\circ} \mathrm{C}$. The certified HSA concentration of SRM 2925 will be determined at NIST by amino acid analysis using isotope dilution LC-MS/MS [7].

The Japanese Society of Clinical Chemistry (JSCC) has proposed highly purified, monomeric, human serum albumin in an aqueous buffer as a secondary reference material for urinary albumin [3]. It was prepared by gelfiltration from commercially-available highly purified monomeric HSA (JSCC in preparation). The assigned value was transferred from HSA in ERM-DA470. The assigned value was $225.1 \pm 9.11 \mathrm{mg} / \mathrm{L}$ (mean \pm expanded uncertainty with coverage factor of 2) when reconstituted with $3.00 \mathrm{~mL}$ of purified water. This monomeric HSA secondary reference material is intended for use as a calibrator or a control in albumin and total protein measurement procedures for urine.

\section{${ }^{15} \mathrm{~N}$-labeled albumin CRM}

The candidate reference measurement procedures for albumin in urine developed at the Mayo Clinic, and under development at NIST, require a series of reagents. These reagents should also be accessible to other reference laboratories. Currently, there are no commercial sources of the ${ }^{15} \mathrm{~N}$-labeled recombinant HSA which is used as the internal standard in the reference measurement procedure. NIST is searching for a commercial laboratory to evaluate approaches for the production of the ${ }^{15} \mathrm{~N}$-labeled recombinant HSA in sufficient quantity and with sufficient isotopic incorporation to be available as a CRM.

\section{Urinary creatinine CRM}

To support the clinical measurement of the ACR in urine samples, NIST has produced SRM 3667 ('Creatinine in Frozen Human Urine'). NIST SRM 3667 was prepared from pooled human urine, collected from a minimum of 10 male and female donors in good health (as self-reported). The urine pool was blended, filtered, and dispensed into amber glass bottles, with a fill volume of approximately $10 \mathrm{~mL}$ each, and stored at $-80^{\circ} \mathrm{C}$. The certification of creatinine in SRM 3667 was performed at NIST using isotope dilution LC-MS in a measurement procedure similar to that used at NIST to certify the concentration of creatinine in serum [8]. The measured concentration of creatinine in SRM 3667 is $5.5 \mathrm{mmol} / \mathrm{L}$ ( $62 \mathrm{mg} / \mathrm{dL})$, and within the typical reference interval. NIST SRM 3667 is expected to be available by the end of 2012 .

\section{Urinary albumin reference measurement procedure}

\section{Materials}

A candidate reference measurement procedure has been developed in the Mayo Clinic Renal Function Laboratory that employs trypsin digestion of whole urine followed by LC-MS/MS [9]. A stock solution of purified human serum albumin (8.74 g/L HSA, \#A3782, Sigma Aldrich, St. Louis, MO, USA) was prepared in water. The concentration was determined by ultraviolet absorption spectroscopy with a molar absorptivity at $280 \mathrm{~nm}$ of $38533 \mathrm{~L} /(\mathrm{mol} \cdot \mathrm{cm})$ [10]. Calibrators were prepared by adding $572 \mu \mathrm{L}$ of the stock solution to a charcoal-stripped urine matrix (BioChemed Services, Winchester, VA, USA) to a final volume of 25 $\mathrm{mL}$ and concentration of $200 \mathrm{mg} / \mathrm{L}$. Serial dilutions were made into the charcoal stripped urine $(12.5 \mathrm{~mL}$ each) to achieve concentrations of 100, 50, 25, 12.5, 6.25, and 3.13 $\mathrm{mg} / \mathrm{L}$. Low (10 mg/L), medium (40 mg/L), and high (106 $\mathrm{mg} / \mathrm{L}$ ) quality controls (QC) were prepared by adding the HSA stock solution to a waste human urine sample with 
endogenous concentration of $0.8 \mathrm{mg} / \mathrm{L}$ albumin. Recombinant ${ }^{15} \mathrm{~N}$-labeled human albumin $\left({ }^{15} \mathrm{NrHSA}\right)$ for use as an internal standard was synthesized in Pichia pastoris as described in the supplemental portion of a previous publication [11]. All calibrators and controls were divided into $0.5 \mathrm{~mL}$ aliquots and frozen at $-80^{\circ} \mathrm{C}$ until used.

Urine samples $(40 \mu \mathrm{L})$ were diluted with ammonium bicarbonate $(0.1 \mathrm{~mol} / \mathrm{L}, 135 \mu \mathrm{L}$, Sigma-Aldrich) to normalize sample $\mathrm{pH}$, and then the ${ }^{15} \mathrm{NrHSA}$ internal standard was added (10 $\mu \mathrm{L}$ of a $161 \mathrm{mg} / \mathrm{L}$ stock). Samples were reduced by the addition of dithiothreitol (final concentration $10 \mathrm{mmol} / \mathrm{L}$, Sigma-Aldrich) for $60 \mathrm{~min}$ at $60^{\circ} \mathrm{C}$, and then alkylated using iodoacetamide $(50 \mathrm{mmol} / \mathrm{L}$, SigmaAldrich) for $30 \mathrm{~min}$ at $25^{\circ} \mathrm{C}$ in the dark. Next L-(tosylamido2-phenyl) ethyl chloromethyl ketone-treated trypsin ( $5 \mu \mathrm{L}$, $1 \mathrm{~g} / \mathrm{L}$ Sigma Aldrich, in $0.1 \mathrm{~mol} / \mathrm{L}$ ammonium bicarbonate) was added and the sample was digested for $1 \mathrm{~h}$ at $37^{\circ} \mathrm{C}$. Finally all samples were acidified with $2 \mu \mathrm{L}$ of concentrated formic acid to result in a final sample volume of $204 \mu \mathrm{L}$.

\section{LC-MS/MS}

Three tryptic peptides that were reproducibly detected in samples of trypsin-digested HSA and distributed throughout the protein were selected for measurement of multiple reaction monitoring (MRM) transitions: ${ }^{42} \mathrm{LVNEVTEFAK}^{51}$, ${ }^{526}$ QTALVELVK $^{534}$ and ${ }^{13}{ }^{\text {DLGEENFK }}{ }^{20}$ (Figure 1). For quantification of: ${ }^{13}$ DLGEENFK ${ }^{20}$ the doubly charged precursor ion at $\mathrm{m} / \mathrm{z} 476.2$ was selected in Q1, and 3 singly charged transitions were monitored in Q3: $\mathrm{m} / \mathrm{z}$ 723.3; 229.1; 201.1;

DAHKSEVAHRFKDLGEENFKALVLIAFAQYLQQCPFEDHVKLVNEVTEFAK TCVADESAENCDKSLHTLFGDKLCTVATLRETYGEMADCCAKQEPERNE CFLQHKDDNPNLPRLVRPEVDVMCTAFHDNEETFLKKYLYEIARRHPYFY APELLFFAKRYKAAFTECCQAADKAACLLPKLDELRDEGKASSAKQRLKC ASLQKFGERAFKAWAVARLSQRFPKAEFAEVSKLVTDLTKVHTECCHGDL LECADDRADLAKYICENQDSISSKLKECCEKPLLEKSHCIAEVENDEMPA DLPSLAADFVESKDVCKNYAEAKDVFLGMFLYEYARRHPDYSVVLLLRLA KTYETTLEKCCAAADPHECYAKVFDEFKPLVEEPQNLIKQNCELFEQLGE YKFQNALLVRYTKKVPQVSTPTLVEVSRNLGKVGSKCCKHPEAKRMPCAE DYLSVVLNQLCVLHEKTPVSDRVTKCCTESLVNRRPCFSALEVDETYVPK EFNAETFTFHADICTLSEKERQIKKQTALVELVKHKPKATKEQLKAVMDD

\section{FAAFVEKCCKADDKETCFAEEGKKLVAASQAALGL}

Figure 1 Amino acid sequence of human serum albumin. Underlined sequences are peptides whose masses have been observed by LC-MS/MS analysis. Those highlighted in red have been reproducibly observed in patient urine samples and used for quantification. for ${ }^{42}$ LVNEVTEFAK $^{51}$ the doubly charged precursor ion at $\mathrm{m} / \mathrm{z} 575.3$ was selected in Q1, and 5 singly charged transitions were monitored in Q3: $\mathrm{m} / \mathrm{z}$ 185.2; 213.2; 595.3; 694.4; 937.5; and for ${ }^{526}{ }^{2 T A L V E L V K}{ }^{534}$ the doubly charged precursor ion at $\mathrm{m} / \mathrm{z} 500.8$ was selected in Q1, and 3 singly charged transitions were monitored in Q3: $\mathrm{m} / \mathrm{z} 700.5$; 587.4; 147.3. Tryptic peptides from the internal standard ${ }^{15} \mathrm{NrHSA}$ were monitored using the same conditions listed above for the native peptides. Quantification was performed by using a Thermo Scientific Aria TLX2 LC system coupled to an Applied Biosystems API 5500 triple-quadrupole mass spectrometer. For each run a total of $20 \mu \mathrm{L}$ of sample digest was injected onto a $50 \times 2.1 \mathrm{~mm}$ TARGA C18 column (Higgins Analytical). Chromatography was performed at a flow rate of $250 \mu \mathrm{L} / \mathrm{min}$. Total run time was $30 \mathrm{~min}$. Mobile phases consisted of solvent A ( $0.1 \%$ formic acid in water) and solvent $\mathrm{B}$ ( $0.1 \%$ formic acid in methanol).

Each final albumin concentration represents the mean value of all transitions for each selected peptide.

\section{Validation to date}

Between-day precision of the candidate reference measurement procedure was evaluated by measuring the seven concentrations of the standard curve over 20 days (Table 1). Within-day precision was validated by measuring the low, medium and high QC material over 20 replicates on the same day (Table 2) by each of three tryptic peptides over 20 different days. All had CVs $<5 \%$. Recovery was evaluated by adding stock HSA to normal patient urine with an endogenous concentration of albumin $0.8 \mathrm{mg} / \mathrm{L}$ to achieve concentrations of 10, 40 and $100 \mathrm{mg} / \mathrm{L}$ (Table 3). Recovery was between $97 \%$ and $108 \%$ with an average of $103 \%$. To assess linearity, waste urine samples containing high concentrations of albumin were serially diluted with $2 \times$ charcoal-stripped urine matrix to produce samples with values within the dynamic range of the assay (Table 4). Linearity, determined by comparing observed values with expected values, was established for dilutions up to 1:4.

A total of 334 human urine samples that covered the range of clinical values (5-1270 $\mathrm{mg} / \mathrm{L}$ ) were measured in duplicate with the LC-MS/MS assay as part of a clinical validation study. This data was analyzed to determine the influence that the number of peptides and resulting transitions that were measured had on the ultimate reproducibility of the value obtained (Table 5). When one peptide and one transition were measured, the $\mathrm{CV}$ was $3.21 \%$. The $\mathrm{CV}$ improved to $2.21 \%$ when two transitions were averaged for each peptide. Reproducibility improved marginally when results from any two peptides were averaged (CV 1.94\%). 


\begin{tabular}{|c|c|c|c|c|c|c|c|c|}
\hline \multirow{2}{*}{$\begin{array}{l}\text { Expected HSA concentration } \\
\text { in urine for calibrator or QC } \\
\text { materials, } \mathrm{mg} / \mathrm{L}\end{array}$} & \multicolumn{2}{|l|}{$\begin{array}{l}\mathrm{HSA}, \mathrm{mg} / \mathrm{L} \\
\mathrm{n}=20 \text { days }\end{array}$} & \multicolumn{2}{|l|}{$\begin{array}{l}\mathrm{HSA}, \mathrm{mg} / \mathrm{L} \\
\mathrm{n}=20 \text { days }\end{array}$} & \multicolumn{2}{|l|}{$\begin{array}{l}\text { HSA, mg/L } \\
n=20 \text { days }\end{array}$} & \multicolumn{2}{|l|}{$\begin{array}{l}\mathrm{HSA}, \mathrm{mg} / \mathrm{L} \\
\mathrm{n}=20 \text { days }\end{array}$} \\
\hline & \multicolumn{2}{|c|}{ LVNEVTEFAK peptide } & \multicolumn{2}{|c|}{ QTALVELVK peptide } & \multicolumn{2}{|c|}{ DLGEENFK peptide } & \multicolumn{2}{|c|}{ Total of 3 peptides } \\
\hline 3.13 & 3.10 & 2.9 & 3.1 & 0.18 & 3.42 & 0.21 & 3.14 & 4.31 \\
\hline 6.25 & 6.07 & 1.4 & 6.33 & 0.22 & 5.47 & 0.17 & 6.14 & 4.70 \\
\hline 12.5 & 12.6 & 1.5 & 12.6 & 0.14 & 11.4 & 0.22 & 12.44 & 3.54 \\
\hline 25 & 25.5 & 0.6 & 24.9 & 0.06 & 24.6 & 0.09 & 24.99 & 1.68 \\
\hline 50 & 52.9 & 1.5 & 50.9 & 0.33 & 49.9 & 0.39 & 51.44 & 1.89 \\
\hline 100 & 95.3 & 1.5 & 95.6 & 0.83 & 102.0 & 0.7 & 96.43 & 2.25 \\
\hline 200 & 202 & 0.7 & 204.2 & 0.37 & 199 & 0.33 & 202.88 & 0.88 \\
\hline 10.8 low QC & 11.3 & 9.9 & 11.5 & 10.2 & 11.8 & 9.5 & 11.55 & 10.48 \\
\hline 40.8 medium QC & 41.5 & 6.8 & 39.95 & 6.4 & 40.6 & 6.4 & 40.28 & 5.97 \\
\hline 106.8 high QC & 105.1 & 4.4 & 101.8 & 3.7 & 103.3 & 4.1 & 103.2 & 4.07 \\
\hline
\end{tabular}

Table 1 Between-day imprecision of the candidate reference measurement procedure for urine albumin over 20 days.

\begin{tabular}{|c|c|c|c|c|c|c|}
\hline \multirow[t]{2}{*}{ Peptides } & \multirow[t]{2}{*}{$\begin{array}{l}\mathrm{HSA}, \mathrm{mg} / \mathrm{L} \\
\mathrm{n}=20 \text { times }\end{array}$} & CV\% & \multicolumn{2}{|l|}{$\begin{array}{l}\text { HSA, mg/L } \\
n=20 \text { times }\end{array}$} & \multirow[t]{2}{*}{$\begin{array}{l}\mathrm{HSA}, \mathrm{mg} / \mathrm{L} \\
\mathrm{n}=20 \text { times }\end{array}$} & CV\% \\
\hline & & Low QC & & $\mathrm{m}$ QC & & High QC \\
\hline LVNEVTEFAK & 12.8 & 1.3 & 44.5 & 4.0 & 111.9 & 2.5 \\
\hline QTALVELVK & 12.9 & 1.1 & 43.4 & 1.3 & 111.5 & 0.6 \\
\hline DLGEENFK & 13.5 & 1.0 & 44.1 & 1.3 & 111.4 & 2.0 \\
\hline $\begin{array}{l}\text { Total of } 3 \\
\text { peptides }\end{array}$ & 13.1 & 2.85 & 44.1 & 4.1 & 11.6 & 2.24 \\
\hline
\end{tabular}

Table 2 Within-day precision evaluated based on the measurement of QC samples (20 replicates).

Using two transitions for each peptide further improved the average CV to $1.80 \%$. Addition of three or more peptides did not improve precision, regardless of the number of transitions used. These results suggest that averaging results from two peptides and two transitions each will provide maximal precision for this measurement procedure.

The albumin concentration indicated by each fragment was also compared across these 334 human urine samples, and the mean $\%$ bias between each fragment pair

\begin{tabular}{llrrr} 
& Peptides & $\begin{array}{r}\text { Expected } \\
\text { mean, mg/L }\end{array}$ & $\begin{array}{r}\text { Calculated } \\
\text { mean, mg/L }\end{array}$ & $\begin{array}{r}\% \\
\text { Recovery }\end{array}$ \\
\hline Sample 1 & LVNEVTEFAK & 10.8 & 10.46 & 97 \\
& QTALVELVK & 10.8 & 10.97 & 102 \\
& DLGEENFK & 10.8 & 11.63 & 108 \\
Sample 2 & LVNEVTEFAK & 40.8 & 41.5 & 102 \\
& QTALVELVK & 40.8 & 41.3 & 101 \\
& DLGEENFK & 40.8 & 42.5 & 104 \\
Sample 3 & LVNEVTEFAK & 100.8 & 104 & 103 \\
& QTALVELVK & 100.8 & 105 & 104 \\
& DLGEENFK & 100.8 & 105 & 104 \\
\hline
\end{tabular}

Table 3 Recovery of HSA (10, 40, and $100 \mathrm{mg} / \mathrm{L})$ spiked into human urine $(0.8 \mathrm{mg} / \mathrm{L})$. assessed by Bland-Altman plots. Indeed, there was a subtle bias such that a peptide fragment towards the amino terminus $\left({ }^{13} \mathrm{DLGEENK}{ }^{20}\right)$ gave values $1.7 \%$ lower than a more centrally located fragment ( ${ }^{138}$ YLYEIAR $\left.{ }^{144}\right)$, while the fragment nearest the carboxyl terminus ( ${ }^{526}$ QTALVELVK $^{534}$ ) gave even lower values (3.6\% lower than ${ }^{138}{ }^{\text {YLYEIAR }}{ }^{144}$, Figure 2). These biases could be consistent with a minor amount of degradation of albumin molecules at both ends while in human urine.

The quantification of intact albumin in urine by trypsin digestion followed by LC-MS/MS could potentially be influenced by smaller endogenous fragments of albumin in the urine sample. To evaluate this possibility, five samples with albumin concentrations within the dynamic range of the assay and five samples with concentrations of albumin more than $1000 \mathrm{mg} / \mathrm{L}$ were selected for study. The waste urine sample $(200 \mu \mathrm{L})$ was loaded on a $10 \mathrm{kD}$ cut Amicon filter to remove small fragments. The retentate (approx. $20 \mu \mathrm{L}$ ) was reconstituted with charcoal-stripped urine matrix $(200 \mu \mathrm{L})$ and concentrated again. Once again the retentate $(20 \mu \mathrm{L}$ now without small fragments $<10 \mathrm{kDa}$ ) was reconstituted with charcoalstripped urine matrix to a final volume of $200 \mu \mathrm{L}$. The concentration of albumin in all five samples before and 


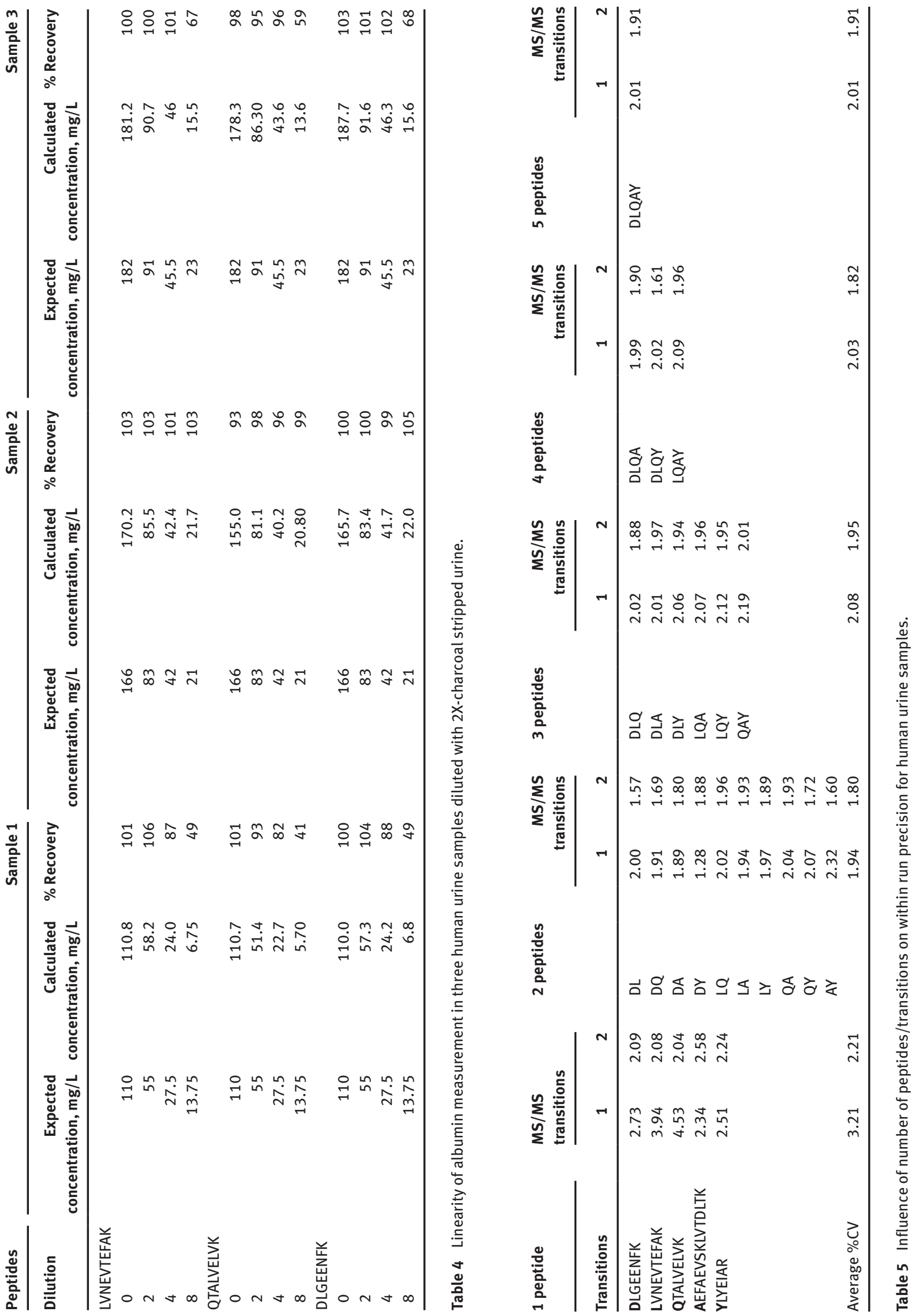




\begin{tabular}{|c|c|c|c|c|c|c|}
\hline & & \multicolumn{5}{|c|}{ Reference fragment } \\
\hline & & ${ }^{13}$ DLGEENK ${ }^{20}$ & ${ }^{42}$ LVNEVTEFAK ${ }^{51}$ & ${ }^{138}$ YLYEIAR $^{144}$ & ${ }^{226}$ AEFAEVSKLVTDLTK ${ }^{240}$ & ${ }^{526}$ QTALVELVK ${ }^{534}$ \\
\hline \multirow{5}{*}{$\begin{array}{l}\text { Comparison } \\
\text { fragment }\end{array}$} & ${ }^{13}$ DLGEENK $^{20}$ & & $-1.1 \%(-0.8,-1.3)$ & $-1.7 \%(-1.3,-2.0)$ & $0.1 \%(0.4,-0.3)$ & $2.0 \%(2.4,1.5)$ \\
\hline & ${ }^{42}$ LVNEVTEFAK $^{51}$ & $1.1 \%(0.8,1.3)$ & & $-0.6 \%(-0.2,-0.9)$ & $1.2 \%(1.5,0.8)$ & $3.1 \%(3.5,2.6)$ \\
\hline & ${ }^{138}$ YLYEIAR ${ }^{144}$ & $1.7 \%(1.3,2.0)$ & $0.6 \%(0.2,0.9)$ & & $1.8 \%(2.2,1.3)$ & $3.6 \%(4.1,3.1)$ \\
\hline & ${ }^{226}$ AEFAEVSKLVTDLTK ${ }^{240}$ & $-0.1 \%(-0.4,0.3)$ & $-1.2 \%(-1.5,-0.8)$ & $-1.8 \%(-2.2,-1.3)$ & & $1.9 \%(2.3,1.5)$ \\
\hline & ${ }^{526}$ QTALVELVK ${ }^{534}$ & $-2.0 \%(-2.4,-1.5)$ & $-3.1 \%(-3.5,-2.6)$ & $-3.6 \%(-4.1,-3.1)$ & $-1.9 \%(-2.3,-1.5)$ & \\
\hline
\end{tabular}

Figure 2 Bias between urinary albumin peptide measurements.

For each of 334 human urine samples, the albumin concentration assessed by each of five trypsin fragments was compared ( 2 transitions each). The mean \% bias $(95 \% \mathrm{Cl}$ ) between each fragment pair was assessed by Bland-Altman plots. There was a subtle bias with the overall lowest values for the fragment nearest the carboxyl terminus.

after concentration was similar (Table 6). Therefore, our studies do not suggest that endogenous albumin fragments interfere with the measurements.

\section{NIST validation of LC-MS/MS assay}

For a reference measurement procedure to be useful and robust, it should be independently validated in a laboratory setting other than the one in which it was developed. As such, the candidate reference measurement procedure developed at the Mayo Clinic is also being implemented at NIST for method validation. The implementation at NIST has maintained the basic measurement strategy of the Mayo LC-MS/MS method; however the NIST measurements have used instrumentation and chromatographic media from manufacturers different than those used by Mayo in order to evaluate the robustness of the methods based on LC-MS. A bilateral measurement comparison of patient samples is planned between NIST and the Mayo Clinic.

In addition to implementing the Mayo Clinic's candidate reference measurement procedure for albumin in urine, NIST has also expanded the method to provide qualitative information on the structure of albumin in urine. Capitalizing on the multiplexed measurement capabilities of modern mass spectrometers, NIST has expanded the LC-MS/MS measurement of three proteolytic peptides from albumin in the Mayo Clinic method to include eight additional proteolytic peptides, 11 peptides in total. The 11 peptides measured include peptides derived from albumin's $\mathrm{N}$ - and C-terminal regions as well as several peptides from the central portion of albumin's protein chain. Measurement of these peptides should provide qualitative information on the 'intactness' of the molecular form of albumin in urine samples to compliment the quantitative information on the concentration of albumin. The ability to evaluate potential molecular heterogeneity of albumin in urine samples while simultaneously quantifying albumin in urine will be very useful in future commutability studies of certified reference materials which will also include measurement of sets of individual patient samples. The qualitative measurement capability will help to identify structural differences in the albumin in patient samples that can be useful in the interpretation of commutability study results and in the assessment of the molecular forms that should be measured by routine clinical laboratory procedures. The number of peptides incorporated into the final candidate reference measurement procedure will be determined once the performance of each of these eight additional peptides has been assessed.

\section{Discussion}

This report describes progress towards a reference measurement system for urinary albumin, a key biomarker of kidney disease. Studies are in progress to assess the current state of the art, including agreement amongst current commercially available routine measurement procedures and commutability of available candidate reference materials. Efforts have also been directed towards development of a candidate reference measurement procedure for urinary albumin, and validation of candidate reference materials for urinary creatinine and albumin. The influence of adsorption of albumin to container surfaces is also being investigated by the working group, although those results are not described here.

Other preanalytic and analytic factors that can also influence use of urinary albumin as a biomarker of renal disease and that may benefit from standardized approaches are summarized below based on the report from the joint working group in 2009 [1].

\section{Biologic/physiologic factors}

Time of urine collection (first morning, second morning, random, or $24 \mathrm{~h}$ ) is important. Twenty-four hour collections may be theoretically ideal, but are not always practical and may be incomplete which will increase variability. Among random urine collections, a first morning void is preferred, since this collection will decrease biologic 


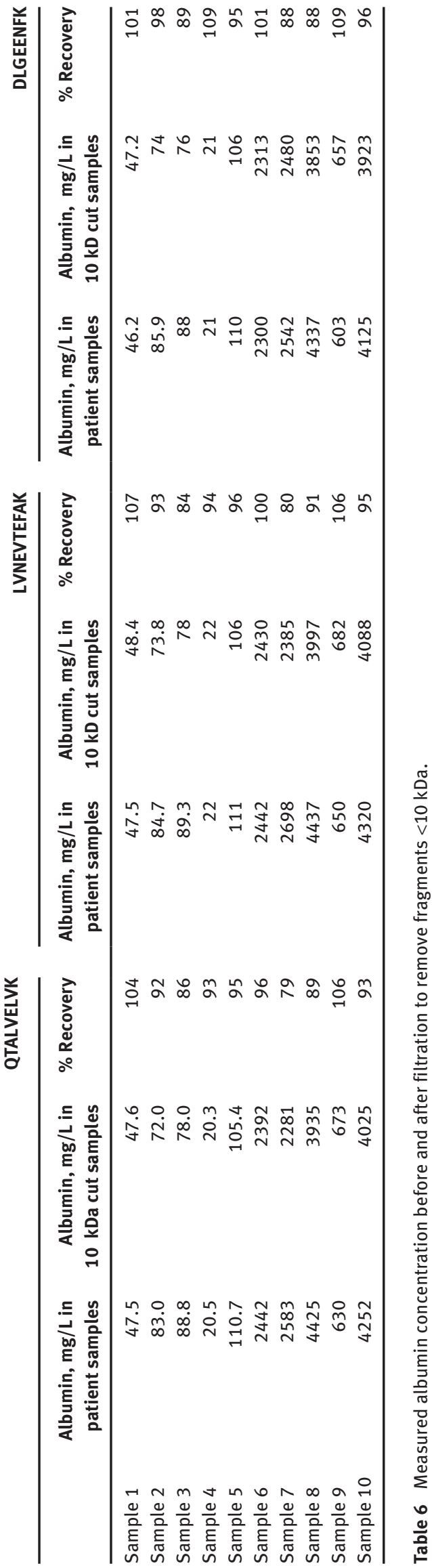

variability [1]. Importantly, different decision limits may be needed for random vs. first morning collections, or other standardized collection time(s) [12].

\section{Stability and interference}

The influence of blood (menstrual or urinary bleeding) seminal fluid and other physiologic contaminants of urine remain to be examined. The molecular forms of albumin in freshly voided urine, and their correlation to kidney disease, has not been fully investigated. Clarification regarding variation of urinary matrix composition and its effects on urinary albumin measurement procedures is needed, including the degree to which albumin degrades under various conditions of storage.

\section{Interpretation}

The ACR varies with age, gender and ethnicity. Decision thresholds suitable for these subgroups need further investigation. The commonly used $30 \mathrm{mg} / \mathrm{g}$ or $3.4 \mathrm{mg} / \mathrm{mmol}$ decision limits may not be adequate for each subgroup in terms of diagnostic sensitivity. Risk of chronic kidney disease and CVD are continuous functions of urinary albumin. The appropriate reference intervals for given populations (e.g., general population or high-risk groups such as diabetes, hypertension or CVD) need to be examined. It would be interesting to examine if age- and gender-specific equations to convert ACR to an estimated albumin excretion rate could be useful to enable using a single reference limit.

\section{Conclusions}

Urinary albumin excretion is a critical marker of kidney disease. Since the initial report of the NKDEP and IFCC joint working group in 2009 [1], much progress toward standardizing measurement of urine albumin has been made, including the development of candidate reference materials and a candidate LC-MS/MS reference measurement procedure.

Acknowledgments: The authors appreciate the contribution of members of the joint working group of the National Kidney Disease Education Program (NKDEP) Laboratory Working Group and the International Federation of Clinical Chemistry and Laboratory Medicine (IFCC) Working Group for Standardization of Albumin in Urine. 


\section{Conflict of interest statement}

Authors' conflict of interest disclosure: The authors stated that there are no conflicts of interest regarding the publication of this article. Research funding: None declared.

\section{References}

1. Miller WG, Bruns DE, Hortin GL, Sandberg S, Aakre KM, McQueen MJ, et al. Current issues in measurement and reporting of urinary albumin excretion. Clin Chem 2009;55:24-38.

2. Zegers I, Keller T, Schreiber W, Sheldon J, Albertini R, BlirupJensen $\mathrm{S}$, et al. Characterization of the new serum protein reference material ERM-DA470k/IFCC: value assignment by immunoassay. Clin Chem 2011;56:1880-8.

3. Itoh Y, Ichihara K, Kishi K, Hosogaya S, Yamada T. Preparation of highly purified monomeric human serum albumin as secondary reference material for standardization of urinary albumin immunoassays. Clin Chim Acta 2012;413:175-81.

4. Baudner S, Bienvenu J, Blirup-Jensen S, Carlström A, Johnson AM, Milfor $\mathrm{A}$, editors. The certification of a matrix reference material for immunochemical measurement of 14 human serum proteins CRM470EUR 15423 EN and 16882 N. Luxembourg: European Community, 1993.

5. Blirup-Jensen S, Johnson AM, Larsen M. Protein standardization V: value transfer. A practical protocol for the assignment of serum protein values from a Reference Material to a Target Material. Clin Chem Lab Med 2008;46:1470-9.

6. Infusino I, Braga F, Valente C, Panteghini M. Commutability of the ERM-DA470k reference material for two assays measuring serum
Employment or leadership: None declared. Honorarium: None declared.

Received May 30, 2012; accepted November 9, 2012; previously published online December 12, 2012

albumin using immunochemical principles. Clin Chem Lab Med 2011;49:1383-4.

7. Bunk DM, Lowenthal MS. Isotope dilution liquid chromatography-tandem mass spectrometry for quantitative amino acid analysis. Methods Mol Biol 2012;828:29-38.

8. Huskova R, Chrastina P, Adam T, Schneiderka P. Determination of creatinine in urine by tandem mass spectrometry. Clin Chim Acta 2004;350:99-106.

9. Seegmiller JC, Barnidge DR, Burns BE, Larson TS, Lieske JC, Kumar R. Quantification of urinary albumin by using protein cleavage and LC-MS/MS. Clin Chem 2009;55:1100-7.

10. Gill SC, von Hippel PH. Calculation of protein extinction coefficients from amino acid sequence data. Anal Biochem 1989;182:319.

11. Singh R, Crow FW, Babic N, Lutz WH, Lieske JC, Larson TS, et al. A liquid chromatography-mass spectrometry method for the quantification of urinary albumin using a novel $15 \mathrm{~N}$-isotopically labeled albumin internal standard. Clin Chem 2007;53:540-2.

12. Witte EC, Lambers Heerspink HJ, de Zeeuw D, Bakker SJ, de Jong PE, Gansevoort R. First morning voids are more reliable than spot urine samples to assess microalbuminuria. J Am Soc Nephrol 2009;20:436-43. 